\title{
Delay-oriented IAB Topology Optimisation
}

\author{
Jasmina McMenamy, Thomas Haustein and Irene Macaluso
}

\begin{abstract}
Integrated access and bachhaul (IAB), introduced in 3GPP Release 16, is expected to be one of the main enablers for dense deployments in mmWave spectrum. The IAB architecture brings in topology changes, which impact networkrelated aspects, such as ensuring robust routing of traffic and load-balancing. While an IAB network should be transparent to the UE, its topology design may affect the end-user performance. Of particular importance in these first releases, hence, is to determine and evaluate optimal IAB topologies. This paper addresses optimal organisation of IAB nodes by considering a number of inter-connected aspects, including maximum number of hops, path-length, load balancing between the IAB-donors and traffic demand in the access network. In that, we focus on one of the parameters most sensitive to the architectural changes in the network - delay. Our analysis relies on the connectivity graph obtained by considering some practical aspects of sectorised beamforming using real $\mathrm{mmWave}$ antenna patterns.
\end{abstract}

\section{INTRODUCTION}

qIn recent years, a huge body of research and standardisation work has emerged, aimed at progressing the enablement of mmWave technology for mmWave mobile communication - see, for example, [1], [2], [3] and references therein. Both academia and industry contributions deal with a whole range of aspects of the mobile communication systems, addressing channel modelling, hardware advances and signal processing, access schemes and user scheduling, cross-layer aspects and others. From a standardisation perspective, 3GPP has completed the first release of New Radio (NR) as part of Release 15 , based on requirements specified in [3]. This is the first mobile communication standard for the mmWave spectrum. The work on further releases of NR continues, and 3GPP is currently working on the specifications of Release 17. One of all NR releases' main objectives is to avail of large swaths of mmWave spectrum by supporting highly-directional, beamoriented communication. While this beam-oriented communication also provides a favourable allocation of radio resources, the mmWave propagation environment still poses significant challenges. It is characterised by the increased path loss and is highly sensitive to blockages caused by various objects, foliage and humans. mmWave links will rely on a dense network of access points (small cells) to tackle these challenges and ensure communication reliability. At sub-6GHz frequencies, a complex interference environment exacerbated by omnidirectional links impedes the performance of these networks. In mmWave bands, the challenging propagation characteristics

Jasmina McMenamy (jasmina.mcmenamy@hhi.fraunhofer.de) is at Fraunhofer Heinrich-Hertz-Institut Berlin, Germany.

Thomas Haustein (thomas.haustein@hhi.fraunhofer.de) is at Fraunhofer Heinrich-Hertz-Institut Berlin, Germany.

Irene Macaluso (macalusi@tcd.ie) is at CONNECT, Trinity College Dublin, Ireland.

This material is based upon works supported by the Science Foundation Ireland under Grant No. 13/RC/2077. and highly directional links become attractive as they significantly reduce interference. However, in urban and denseurban settings, which these deployments target, installation costs, potential planning objections and delays in laying fibre to the site make fibre-connectivity to each of these nodes quite challenging. Hence, one of the main prerequisites of dense mmWave deployments is the self-backhauling capability of access points. In that respect, 3GPP's Integrated Access and Backhaul fully address these requirements. It should be noted that IAB is specified to support both sub- $6 \mathrm{GHz}$ and mmWave spectrum. It supports in-band and out-band communication, enabling flexible use of spectrum between access and backhaul. Both - half-duplex and full-duplex are supported. Compared to the previous generation of relays in LTE [4], IAB enables having more than a single relay node and hence, supports true multi-hop communication - crucial in mmWave environment. Besides, IAB nodes are designed to facilitate a new type of base station architecture with a split/disaggregated protocol stack between a distributed unit (DU) with lower layers of the protocol stack and a central unit (CU) with centralised higher layer protocol functions. Thus, the IAB nodes house a DU, and a UE-like mobile termination part (MT), as will be discussed later, while the nodes that provide both - DU and CU functionality are referred to as IAB donors. With the completion of standardisation of the first IAB release as a part of Release 16, the operators will look to introduce these standardised solutions into their networks, particularly to progress mmWave deployments. Clearly, the users' performance will depend not only on the radio access network but also on various aspects of the wireless backhaul network, such as physical deployment and the ability to adapt its topology to the propagation challenges or the load in the network. In our previous work [5], we analysed the Madridgrid based topology of mmWave backhaul nodes with real antenna patterns. The focus of that work was to minimise the number of fibre-connected nodes with a constraint on the number of hops, taking into account practical limitations of the nodes, such as the number of radiofrequency (RF) chains. In this work, we consider IAB topology optimisation, focusing on the important aspects of delay and load-balancing in the backhaul part while also considering the traffic load generated in the access network. Hence, the following subsection provides a reader with an overview of relevant work in this area and summarises this work's contributions.

\section{A. Related work \& our contribution}

IAB network has gained much attraction from research and industry alike as it enables fully integrated wireless access and backhaul connectivity while availing of large swaths of mmWave spectrum. In [6], the authors summarize key aspects 
of Release 16 specifications and outline Release 17 IAB enhancements. Using a finite stochastic geometry model for both - IAB nodes and UEs, they provide performance analysis of IAB nodes in both dense and suburban areas. The IAB nodes make a network of small cells, supported by a few macro base stations. The authors compare the UE's service coverage rate between the small-cell network with IAB nodes only and a hybrid small-cell network of IAB and fibre-backhauled nodes. They also study the robustness of IAB networks and how various deployment conditions such as blockage, tree foliage, rain and antenna height/gain affect coverage rate. Various distributed mmWave backhaul path-selection policies are analysed in [7], with policies mainly using link quality and SNR as a metric, as well as achievable rate. The policies are also designed to incorporate a function that biases the link selection towards nodes with the wired backhaul to reduce the number of hops. In [8], the same authors extend the work by analysing user throughput and end-to-end latency using some of the designed path-selection policies. As observed by the authors, these distributed policies can be used to adapt the IAB topology in case of link failures or include a new IAB node in an uncoordinated fashion.

In our work, we propose a centralised approach to optimise different aspects of the IAB network, including selecting the IAB-donors, the backhaul paths, and balancing the traffic load of IAB-donors. Centralised approaches, such as the ones proposed in this work, and distributed mechanisms such as the ones presented in [7], [8], can work in tandem to meet network-wide objectives while also adapting to local, sudden changes in the environment (e.g. in case of blockage events). The network optimisation we propose could run within a network controller that adapts to changes that require a response time of a second or more (e.g. load balancing), such as, for example, Non- Real-Time (Non-RT) RAN Intelligent Controller (RIC) in the system's orchestrator in the emerging OPEN RAN architecture.

In [9], the authors analyse topology formation (activation of links) and formulate it as a graph optimisation problem that maximises the lower bound of the network capacity, using a combination of deep reinforcement learning and graph embedding. We adopt a different approach for building the connectivity graph, which is subsequently used by the optimisation presented in this paper. Our connectivity graph is obtained by considering some practical aspects of sectorised beamforming using mmWave antenna patterns at $73 \mathrm{GHz}$. We then analyse traffic demand in the access network, load balancing across IAB-donors, the maximum number of hops, and the selected routes' path length to optimally select the association between IAB-donors and IAB-nodes, and the path connecting them. The authors in [10] investigate joint resource allocation and relay selection in a multi-hop IAB network to maximise the geometric mean of UE rates. They compare different optimisation variants, considering Reference Signal Receive Power (RSRP)-based spanning tree and mesh IAB network, including the impact of load-balancing in the access network. Minimising the number of donors is a common objective in [10] and our work. However, the authors there incorporate a constraint on the UE demand in the access network, while this work includes the constraint on the maximum number of hops, informed by delay considerations for eMBB traffic. For the path selection, they propose an approach based on flow maximisation, while, as stated earlier, in this work we consider traffic demand in the access network, load balancing across IAB-donors, the maximum number of hops, and the path-length of the selected routes.

In summary, the contributions of this work are as follows:

1) Formulation of the optimal IAB network topology as a problem that deals with several interconnected features, which include the maximum number of hops, pathlength/ delay, load balancing in the IAB donors and traffic demand in the access network;

- The optimisation model also considers a condition whereby an IAB node can only relay traffic towards the same IAB-donor the node is assigned to, i.e. the traffic destined for different IAB donors does not traverse the same IAB node, which is in line with Release 16 specifications;

2) Illustrative analysis of delay, which informs our choice of the maximum number of hops for the evaluation of the adopted approach;

3) Extensive evaluation based on the connectivity graph that incorporates aspects of sectorised beamforming using real mmWave antenna patterns generated at $73 \mathrm{GHz}$.

The article is organised in the following way: Section II provides an overview of the IAB architecture and presents illustrative delay analysis on the radio interface using 3GPP studies as a basis. Section III presents the optimisation models that deal with the IAB-donor selection and different objectives to organise IAB nodes' network optimally. Results and Conclusion are given in Sections IV and V, respectively.

\section{DEPLOYMENTS WITH IAB NODES}

As stated in the previous section, IAB enables multihop wireless relaying, particularly targeting deployments in mmWave networks. It has been designed to facilitate $\mathrm{gNB}$ split/disaggregated, providing deployment flexibility to move functions between DU and CU. The gNB split architecture has been adopted in Release 15 [11], placing the Radio Link Control (RLC), Medium Access Control (MAC) and PHY layer in the DU while Packed Data Convergence Protocol (PDCP) and Radio Resource Control (RRC) layers are located in the CU. CU provides a subset of gNB functions, such as mobility control, session management, transfer of data to the core network, and controls multiple DUs' operations. The CU and DU communicate via the F1 interface - control plane data is carried via F1-C and user plane data via the F1-U interface. The IAB node's architecture fits well with the split architecture and comprises two parts - IAB-DU and IAB-MT. IAB-DU provides the gNB DU functionality, whereas IAB-MT supports a subset of the UE functionality, which includes, e.g. PHY layer, Layer 2, RRC protocol and Non-Access Stratum. IABMT, hence, enables a connection to another DU, the IABdonor $\mathrm{CU}$ and the core network.

IAB topology falls under the directed acyclic graph (DAG), which means there are no cycles (loops), and the graph has 


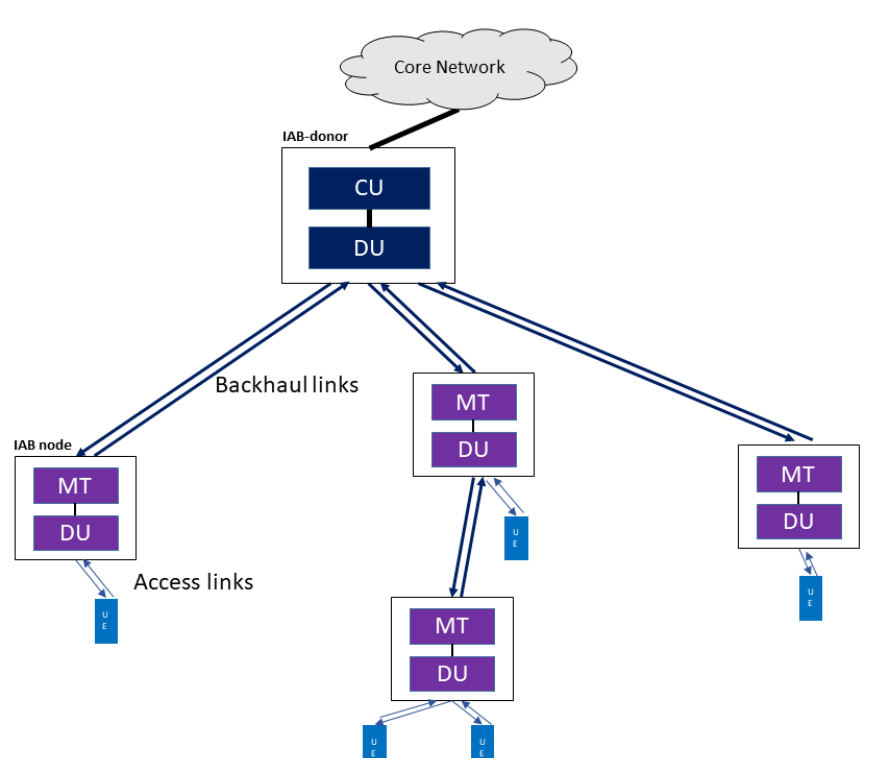

Fig. 1. IAB network hierarchy. At the top of the hierarchy is IAB-donor, which features DU and CU. Note that an IAB-donor may feature multiple DUs as well as separate control and user-plane functions.

a direction. DAG is also hierarchical in that an IAB node connects upstream to its parent, which may also be an IAB donor. The nodes lower in the hierarchy are referred to as child nodes, including UEs - Figure 1 depicts an example of an IAB network. To enable data transfer, routing, flow control and support for QoS in the backhaul network, the Backhaul Adaptation Protocol (BAP) layer is introduced to the protocol stack, above the RLC layer. The IAB-donor-CU configures paths in the IAB network, whereby the configuration includes a unique BAP routing-id and the next-hop BAP address. In terms of topology support, Release 16 envisages that an IAB node may be dual-connected, i.e. it may have multiple parents. The routes may be used for load-balancing and increased reliability, but they need to be underneath the same IABdonor-CU. Route redundancy may also be used as a backup option. In the ongoing work as a part of Release 17, some of the main objectives include features that improve reliability, load-balancing and topology management. For example, one of the major Release 17 objectives is to address inter-IAB-donor IAB-node migration. Hence, it can be expected that some of these features will be more prevalent in later deployments.

\section{A. Delay considerations}

One of the most important aspects of the IAB-based multihop backhauling is its transparency to the UE. This means that UE should not be aware of how it is connected to the core network and Internet cloud - through a multi-hop IABbased backhaul or a conventional gNB node. In providing such transparency, particularly important is the delay introduced by multi-hop backhauling. While the standard does not specify the maximum number of hops in the IAB network, each hop adds processing and propagation delay. These aspects need to be considered in analysing user- and control-plane data delay requirements, particularly given various deployment scenarios. In 5G, the user-plane latency requirement, i.e., the

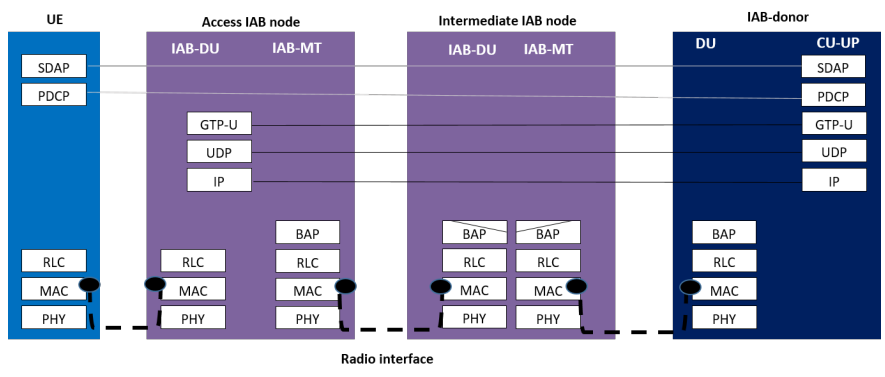

Fig. 2. User-plane protocol stack in the IAB network. Every backhaul hop, besides radio interface and RLC delay, introduces the BAP layer delay. Delay on the F1 interface between access DU and CU as well as PDCP delay at the UE and IAB-donor-CU also need to be taken into when calculating the overall RAN delay.

radio network's contribution to the time from when the source sends a packet to when the destination receives it, ranges between 0.5 and $4 \mathrm{~ms}$ (one way), depending on the type of $5 \mathrm{G}$ services [12].

For illustration, let us look at the impact of an IAB network on user-plane radio interface delay. The user-plane latency in the radio access network - between UE and gNB, is defined as the time it takes to successfully deliver an application layer packet/ message from the radio protocol layer 2/3 SDU ingress point to the radio protocol layer 2/3 SDU egress point via the radio interface in either direction. In other words, the user plane latency is the latency from the time when the transmitter's PDCP receives an IP packet to the time when the receiver's PDCP successfully receives the SDU and delivers the packet to the upper layer. This delay includes PDCP/RLC processing delay by the UE and gNB and over-the-air interface delay. In split architecture, there is also a delay on the interface between DU and CU itself, while the gNB node processing delay is split between the DU and CU. According to [13], the minimum requirements for user-plane latency (from source to destination) are $4 \mathrm{~ms}$ for eMBB and $0.5 \mathrm{~ms}$ for URLLC traffic. These requirements are transparent to all RAN architecture options. In this analysis, we focus on the air-interface delay component in an IAB network, taking the uplink case as an example.

Analogous to the evaluation in $[14]^{1}$, for each hop on the $\mathrm{UL}$, the air-interface delay between a UE and a gNB can be expressed as

$$
T_{\text {airDelayHop }}=t_{\mathrm{MT}, \mathrm{tx}}+t_{\mathrm{FA}}+t_{\mathrm{UL}_{\mathrm{duration}}}+t_{\mathrm{DU}, \mathrm{rx}}+n * t_{\mathrm{rtx}}
$$

Here, $t_{\mathrm{MT}, \mathrm{tx}}$ is the Tx processing delay by the MT, $t_{\mathrm{FA}}$ is the frame alignment time and the waiting time for next available $\mathrm{UL}$ slot, $t_{\mathrm{UL}_{\text {duration }}}$ is e.g. the length of one-slot in a slotted transmission and $t_{\mathrm{DU}, \mathrm{rx}}$ is the Rx processing delay on the DU side. Further, $t_{\mathrm{rtx}}$ is the time required for one re-transmission, and $\mathrm{n}$ is the number of re-transmissions.

Air interface delay includes propagation delay, which may be considered negligible as it depends on the speed of propagation (speed of light in the wireless environment) and the distance, which is in the order of hundred meters.

\footnotetext{
${ }^{1}$ See Table 5.7.1.1.2-1 in [14].
} 
In the delay analysis from [14], different Time Division Duplex (TDD) UL/DL frame structures are considered, also in mmWave spectrum. The evaluation there considers transmission error probability of 0 and 0.1 as well as grant-free transmission, i.e. a transmission scheme without scheduling request towards a gNB and a subsequent dynamic grant to the UE. With these parameters ${ }^{2}$, in most cases, one-way radio latency is $0.59 \mathrm{~ms}$ for error-free transmission and $0.67 \mathrm{~ms}$ for the error probability of 0.1 . Applying these figures to the IAB nodes, and given the eMBB user-plane latency target of $4 \mathrm{~ms}$, we can conclude that the maximum number of hops to meet the target is six (6) for error-free transmission, that is five (5) hops for error probability of 0.1. It should be noted that delay components for BAP and RLC/PDCP processing or the IAB-donor transport delay between its $\mathrm{DU}$ and $\mathrm{CU}$ need to meet the remaining delay budget. In $[15]^{3}$, the userplane latency for the uplink data with a configured grant was considered. The report concludes that $0.82 \mathrm{~ms}$ for the first transmission, $1.6 \mathrm{~ms}$ for the first re-transmission, $2.3 \mathrm{~ms}$ for the second re-transmission and $3.1 \mathrm{~ms}$ for the third re-transmission are suitable to meet the user-plane latency for eMBB traffic. With these latency figures and the assumptions that IAB nodes introduce the same processing delay, the maximum number of hops to meet the latency target of $4 \mathrm{~ms}$ in an IAB network is now reduced to four (4), provided, again, that sum of all other delay components can meet the remaining delay budget. Should the re-transmissions be required, the constraint on the number of hops in this case becomes significantly tighter and it halves for the first re-transmission, illustrating the importance of quality of links in meeting the latency requirements.

\section{IAB TOPOLOGY OPTIMISATION}

This section describes our approach to optimising and analysing an IAB network, focusing on the backhaul aspects, while also taking into account the traffic in the access network. The approach consists of two distinct parts: the first stage addresses an optimum IAB-donor selection corresponding to deployment, while the second part addresses the optimal organisation of IAB nodes with respect to different aspects of the IAB network, such as the backhaul path-length and the IAB-donor load distribution. Both stages rely on a graphbased representation of the IAB network, which we refer to as the connectivity graph. The connectivity graph is the same as in [16], where we considered realistic deployment topology using the Madrid grid layout for the wireless backhaul performance analysis in mmWave band, at $73 \mathrm{GHz}$. The connectivity graph was obtained by further considering nodes' hardware constraints, such as the number of RF chains and the realistic channel model that incorporated real beamforming gains, as per the details outlined in the next section. First, however, we address the IAB-donor selection and optimum organisation of IAB nodes.

\footnotetext{
${ }^{2}$ We used the examples with sub-carrier spacing of $120 \mathrm{KHz}$, with a regular transmission time interval length of 14 OFDM symbols.

${ }^{3}$ See Table 27.
}

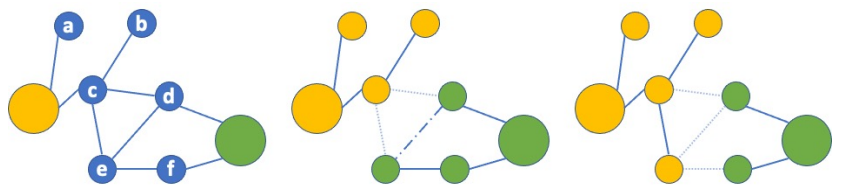

Fig. 3. The left-hand side graph represents a multi-hop network with two IAB-donors (yellow and green larger nodes), six IAB nodes (smaller blue nodes) and their connections. The graphs in the centre and right-hand side represent two possible partitions of the network. In line with Release 16, links connecting two IAB nodes that are assigned to different IAB-donors are not used and are shown as dashed lines in the figure. The dash-dotted line in the centre figure connecting nodes $e$ and $d$, which are assigned to the same IAB donor, is a redundant route.

\section{A. IAB-donor selection}

Considering that IAB-donors provide full protocol stack and physical interfaces to the core network, their infrastructure requirements are significantly greater - they require wired connectivity to the core network, as well as more processing power, space and cooling. Mechanisms to determine how many IAB-donors will be deployed and where they should be located will depend on a variety of considerations. Among them, the minimisation of the number of IAB-donors is likely to be one of the driving factors. Similarly to our previous work [16], in this section, we model the IAB-donor selection problem as a minimum hitting set problem, which allows us to minimise the number of IAB-donors while guaranteeing that each node in the network can reach at least one IAB-donor with less than a maximum number of hops.

Let us denote by $A_{n}$ the set of nodes in the connectivity graph that are reachable by node $n$ with at most $H$ hops. Given the set of nodes $N$ and the set $A$ of all $A_{n}$, the objective is to find the smallest subset of $N$ which intersects every $A_{n}$. The resulting minimum hitting set will cover all the nodes in $N$, i.e. it allows to reach each node in $N$ from the selected hitting set (IAB-donors) with a path of at most $H$ hops, and is formulated as:

$$
\begin{array}{ll}
\min _{n \in N} y_{n} & \\
\text { s.t. } & \forall \alpha \in A \\
\sum_{n \in \alpha} y_{n} \geq 1 & \forall n \in N . \\
y_{n} \in\{0,1\} &
\end{array}
$$

Each element of $A$, denoted by $\alpha$ in Eq. (2), is the set of nodes that are within $H$ hops of a node in $N$. By forcing the selection of at least one node for each of these sets, the constraint in Eq. (2) ensures that each node in $N$ is at most $H$ hops away from a donor.

\section{B. IAB nodes optimal organisation}

In this sub-section, we present solutions to optimally organise the IAB nodes in a multi-hop wireless network for a given deployment of IAB-donors and a maximum number of hops. We consider different optimisation objectives, such as IAB-donors load-balancing and backhaul path length. These solutions enforce the requirement that an IAB node can only 
relay traffic towards the same IAB-donor the node is assigned to, i.e. the traffic does not traverse the same IAB node destined for different IAB donors. This simplifies the routing mechanism and is also in line with Release 16 specifications. Also, only a single path/route between a donor IAB-donor and an IAB node is used at a time, although different routes may exist. In other words, multi-connectivity towards the same IAB-donor is supported as a back-up option.

The optimisation problems in this work address separately the objectives of maximising the load-balancing between different IAB-donor and minimising the path-length, as well as the combination of these objectives. Several formulations are possible, for which the notation is provided in Table I.

The first formulation addresses the aspect of load-balancing and is given by equations (2-6). The combination of the objective function Eq. (3) and constraints Eq. (4) and Eq. (5) minimises the maximum of absolute deviations between the load assigned to each IAB-donor. Constraint Eq. (6) ensures that for each IAB node, one path must be selected while Eq. (7) ensures that two paths headed for different IAB-donors cannot intersect. This means that each IAB node is connected to only one IAB-donor, and the links connecting nodes assigned to different donors cannot be used. For example, if node $c$ in Fig. 3 is assigned to the yellow IAB-donor, node $b$ can only be assigned to the yellow IAB-donor, despite the existence of a path between $b$ and the green IAB-donor.

Balancing the load is only one of the possible objectives to organise IAB nodes. Despite having limited the number of allowed hops in the network, another interesting solution is the partition of the nodes that minimises the overall network delay, estimated in Eq. (8) as the sum of all selected paths lengths.

Finally, a combination of the two previous formulations is given in the third formulation. Here the objective function Eq. (11) is the same as Eq. (8), i.e. it minimises the sum of all selected paths lengths. $z *$ in constraints Eq. (12) and Eq. (13) is the optimal value of Eq. (3). Hence, constraints Eq. (12) and Eq. (13) ensure that the maximum of absolute deviations between the load assigned to each IAB-donor is at most $f(z *)$. If $f(z *)=z *$, the solution to this third formulation is also optimal for the first formulation, but also minimises the sum of all selected paths lengths. In other words, among all possible optimal solutions to equations (3)-(7), the third formulation with $f(z *)=z *$ selects the one corresponding to the smallest sum of all selected paths lengths. If $f(z *)>z *$, the constraint on the absolute deviations is relaxed, which may result in a lower value of the overall total paths lengths.

\section{RESUlts}

As outlined in the previous section, the network deployment in this work is the same as in [16], where we considered Madrid grid layout. This model is regarded as a compromise between the Manhattan model and site-specific urban environment models, with rectangular buildings of different heights. All the nodes in our work are deployed along the streets every 63 or 75 meters, whereas two macro base stations, as per the assumptions in [17] are deployed on
TABLE I

NOTATION USED IN OPTIMISATION FORMULATIONS

\begin{tabular}{|l|l|}
\hline Symbol & Definition \\
\hline \hline$C$ & Set of IAB-donors \\
\hline$D$ & Set of IAB nodes \\
\hline$L$ & Set of admissible path lengths \\
\hline$P_{d, c, l}$ & $\begin{array}{l}\text { Set of paths between node } d \in D \text { and } \\
\text { node } c \in C \text { of length } l \in L\end{array}$ \\
\hline$p_{d, c, l, r}$ & $\begin{array}{l}\text { the } \mathrm{r} \text {-th path in the connectivity graph } \\
\text { between node } d \in D \text { and node } c \in C \\
\text { of length } l\end{array}$ \\
\hline$\delta\left(p_{d, c, l, r}, p_{d^{\prime}, c^{\prime}, l^{\prime}, r^{\prime}}\right)$ & $\begin{array}{l}\text { Binary indicator. It is 1 iff the two } \\
\text { paths } p_{d, c, l, r} \text { and } p_{d^{\prime}, c^{\prime}, l^{\prime}, r^{\prime} \text { intersect }} \\
\text { and } c \neq c^{\prime}\end{array}$ \\
\hline$t_{i}$ & Traffic load of node $i$ \\
\hline$T=\sum_{i \in D \cup C} t_{i}$ & Total traffic in the network \\
\hline$x_{d, c, l, r}$ & $\begin{array}{l}\text { Binary variable which is equal to 1 if } \\
p_{d, c, l, r} \text { is selected and } 0 \text { otherwise }\end{array}$ \\
\hline
\end{tabular}

rooftops, providing wireless connectivity to the neighbouring nodes. While the nodes located in the middle of a street have links along and across the street, nodes on the corners also have diagonal links. The work considers only Line-of-Sight (LoS) propagation state. In total, there are 98 nodes, two of which are macro base-stations. The mmWave links are realised using fixed beamforming with high-gain antenna arrays, where each antenna array covered a $90^{\circ}$ sector in the horizontal plane. The antenna array synthesised beams with a half-power beamwidth of $6^{\circ}$. All links except for the links from macrocells are realised in the horizontal plane. The antenna arrays were designed using CST Microwave Studio software [18]. The adopted channel model is the close-in free space pathloss channel model with reference distance, according to [19]. Table II outlines the main parameters used in our Matlab-based simulations.

TABLE II

SIMULATION SETUP USED TO OBTAIN CONNECTIVITY GRAPH

\begin{tabular}{|l|l|}
\hline \hline Operating frequency & $73 \mathrm{GHz}$ \\
\hline Bandwidth (B) & $1 \mathrm{GHz}$ \\
\hline \hline Macro \& BN/BGW height & $52 \mathrm{~m} \mathrm{\&} 10 \mathrm{~m}$ \\
\hline $\begin{array}{c}\text { 2-D distance between the } \\
\text { nodes along the grid }\end{array}$ & $12 \mathrm{~m}, 63 \mathrm{~m} \mathrm{\&} \mathrm{75m}$ \\
\hline \hline $\begin{array}{c}\text { Half-power beamwidth } \\
\text { (HPBW) - azimuth }\end{array}$ & $6^{\circ}$ \\
\hline \hline Tx power (beam) & $10 \mathrm{dBm}$ \\
\hline \hline
\end{tabular}

For the solution to the optimisation models presented in Section III, we used the CPLEX Concert Library.

Let us first look at the problem of IAB-donor selection. Considering, for example, $H=4$ hops in the backhaul network, based on our results in Section II, the optimal solution to Eq. (2) in case of the Madrid grid-pattern deployment shown in Fig. 4 is to deploy four IAB-donors along the perimeter of the considered area and as far away as possible from each other. However, if we take into account that a macro cell will most likely be designated as an IAB-donor, we can modify the minimum hitting problem Eq. (2) so that the two macrocells (shown in the figure as diamond-shaped markers) are selected as IAB-donors. The optimal solution 


$$
\begin{aligned}
& \min \quad z \\
& -z+\sum_{d \in D} \sum_{l \in L} \sum_{r=1}^{\left|P_{d, c, l}\right|} t_{d} x_{d, c, l, r} \leq \frac{T}{|C|} \\
& -z-\sum_{d \in D} \sum_{l \in L} \sum_{r=1}^{\left|P_{d, c, l}\right|} t_{d} x_{d, c, l, r} \leq-\frac{T}{|C|} \\
& \sum_{c \in C} \sum_{l \in L} \sum_{r=1}^{\left|P_{d, c, l}\right|} x_{d, c, l, r}=1 \\
& \delta\left(p_{d, c, l, r}, p_{d^{\prime}, c^{\prime}, l^{\prime}, r^{\prime}}\right)\left(x_{d, c, l, r}+x_{d^{\prime}, c^{\prime}, l^{\prime}, r^{\prime}}\right) \leq 1 \\
& \forall d, d^{\prime} \in D, \forall c, c^{\prime} \in C, \forall l, l^{\prime} \in L, \\
& \forall r \in\left\{1,2, \ldots,\left|P_{d, c, l}\right|\right\}, \forall r^{\prime} \in\left\{1,2, \ldots,\left|P_{d^{\prime}, c^{\prime}, l^{\prime}}\right|\right\}
\end{aligned}
$$

$$
\begin{aligned}
& \min \sum_{c \in C} \sum_{d \in D} \sum_{l \in L} l \sum_{r=1}^{\left|P_{d, c, l}\right|} x_{d, c, l, r} \\
& \sum_{c \in C} \sum_{l \in L} \sum_{r=1}^{\left|P_{d, c, l}\right|} x_{d, c, l, r}=1 \\
& \delta\left(p_{d, c, l, r}, p_{d^{\prime}, c^{\prime}, l^{\prime}, r^{\prime}}\right)\left(x_{d, c, l, r}+x_{d^{\prime}, c^{\prime}, l^{\prime}, r^{\prime}}\right) \leq 1
\end{aligned}
$$

$$
\begin{array}{r}
\forall d \in D \\
\forall d, d^{\prime} \in D, \forall c, c^{\prime} \in C, \forall l, l^{\prime} \in L, \\
\forall r \in\left\{1,2, \ldots,\left|P_{d, c, l}\right|\right\}, \forall r^{\prime} \in\left\{1,2, \ldots,\left|P_{d^{\prime}, c^{\prime}, l^{\prime}}\right|\right\}
\end{array}
$$

$$
\begin{aligned}
& \min \sum_{c \in C} \sum_{d \in D} \sum_{l \in L} l \sum_{r=1}^{\left|P_{d, c, l}\right|} x_{d, c, l, r} \\
& \sum_{d \in D} \sum_{l \in L} \sum_{r=1}^{\left|P_{d, c, l}\right|} t_{d} x_{d, c, l, r} \leq \frac{T}{|C|}+f\left(z^{*}\right) \\
& -\sum_{d \in D} \sum_{l \in L} \sum_{r=1}^{\left|P_{d, c, l}\right|} t_{d} x_{d, c, l, r} \leq-\frac{T}{|C|}+f\left(z^{*}\right) \\
& \sum_{c \in C} \sum_{l \in L} \sum_{r=1}^{\left|P_{d, c, l}\right|} x_{d, c, l, r}=1 \\
& \delta\left(p_{d, c, l, r}, p_{d^{\prime}, c^{\prime}, l^{\prime}, r^{\prime}}\right)\left(x_{d, c, l, r}+x_{d^{\prime}, c^{\prime}, l^{\prime}, r^{\prime}}\right) \leq 1
\end{aligned}
$$

to this modified minimum hitting problem is to deploy five IAB-donors, two of which are the two macrocells. Among all the optimal solutions, for our subsequent analysis in this section, we chose a solution that corresponds to (i) IAB-donors deployed along the south-north roads where the macrocells are located $^{4}$; (ii) IAB-donors as widely dispersed as possible.

Fig. 4 and Fig. 5 both depict the deployment scenario with IAB nodes deployed in the Madrid grid-pattern, corresponding to the solution of Eq. (3). Fig. 4 depicts the IAB nodes partitions in case of uniform traffic demand per node, whereas Fig. 5 depicts the case where traffic demand is unevenly distributed among the IAB nodes with a few selected nodes (enclosed in the red rectangle) having twice the load compared to the others. Such an assumption help us identify the effect the IAB node demand distribution has on the optimal IAB-donors

\footnotetext{
${ }^{4}$ Deployments along roads are realistic as they minimise fibre deployment costs.
}

load distribution resulting from equations (3)-(7), which is depicted in Fig. 6. Here, the blue bars correspond to the uniform IAB node traffic demand, whereas the red bars refer to the uneven traffic demand case, as per Fig. 5. Hence, for the IAB-donors to handle the same traffic demand, the number of associated IAB nodes per IAB-donor will be differently distributed. With uniform traffic demand, the IAB nodes are distributed as uniformly as possible across all IAB donors. In the case of non-uniform traffic demand, there is one IAB-donor (i.e. the orange IAB-donor in Fig. 5) that manages seventeen IAB nodes, five of which handle twice the demand of other nodes, which is equivalent to managing in total twenty-two IAB nodes. In Fig. 5, as a result, the IAB donors deployed on the easternmost road are assigned a smaller number of nodes, and the IAB donors on the westernmost road have to compensate by managing more IAB nodes. For example, two nodes previously assigned to the light green IAB-donor in Fig. 


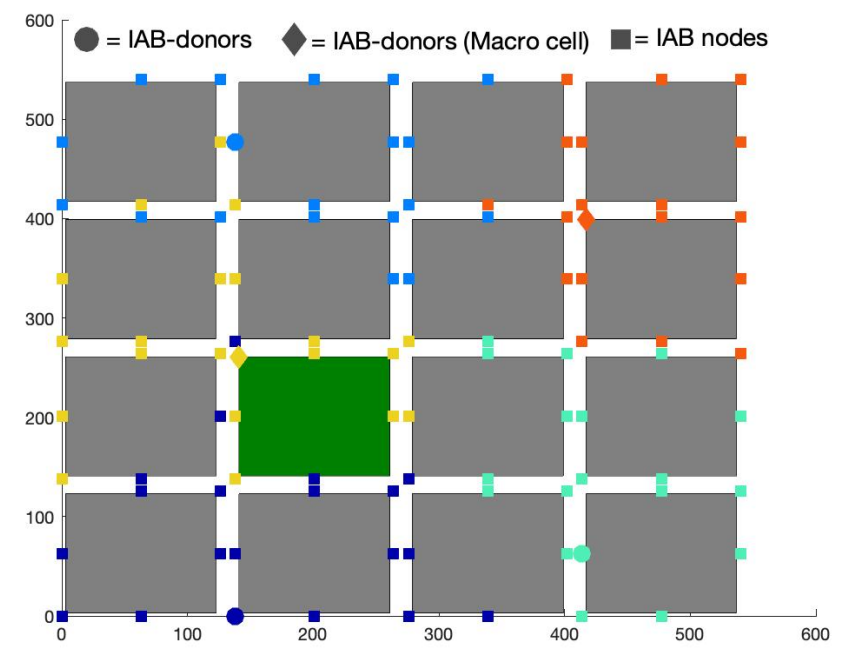

Fig. 4. Partition of the IAB nodes corresponding to the uniform traffic demand for the Madrid-grid deployment with 5 IAB-donors, two of which are macrocells (diamond markers). The remaining three donors are shown as circles. Each IAB-donor is shown in a different colour. IAB nodes are shown as coloured squares. The colour indicates the IAB-donor to which an IAB node is associated.

4, are now assigned to the yellow IAB-donor in Fig. 5.

Fig. 7 shows the path length distribution corresponding to the formulation in equations (8)-(10). More than $67 \%$ of the IAB nodes can reach their assigned IAB-donor in 1 or 2 hops, and only 6 out of 93 IAB nodes require 4 hops to reach the IAB-donor. Here, the results are the same for uniform and non-uniform traffic demand as there is no constraint on the IAB-donor load.

Fig. 8 depicts the effect the IAB-donor load constraint has on the path-length distribution, corresponding to the formulations in Eq. (3) and Eq. (11). The formulation in Eq. (3) ensures that the load among donors is as equally distributed as possible. The formulation in Eq. (11) relies on a combination of load and path length to determine the optimal solution. In particular, if $f\left(z^{*}\right)=z^{*}$ a solution to Eq. (11) is also a solution to Eq. (3), and in particular it is a solution that also minimises the overall path lengths. The greater the value of $f\left(z^{*}\right)$, the looser is the constraint on IAB-donor load balancing and the resulting path-length distribution is then approaching the distribution Fig. 7, for both uniform and non-uniform traffic demand.

\section{CONCLUSION}

IAB nodes will be inherent to mmWave network deployments. Such deployments not only have to provide mechanisms for tackling sudden blockages and ensuring reliable backhaul and access links but also need to be organised to minimise delay in the network and ensure load-balancing while catering for varying traffic demand in different parts of the access network. These are some of the aspects this work addresses. In particular, we formulate the IAB network's optimal organisation as a problem that deals with a number of interconnected features, which include a maximum number of hops, path-length/delay, load balancing between the IABdonors and traffic demand in the access network. We provide

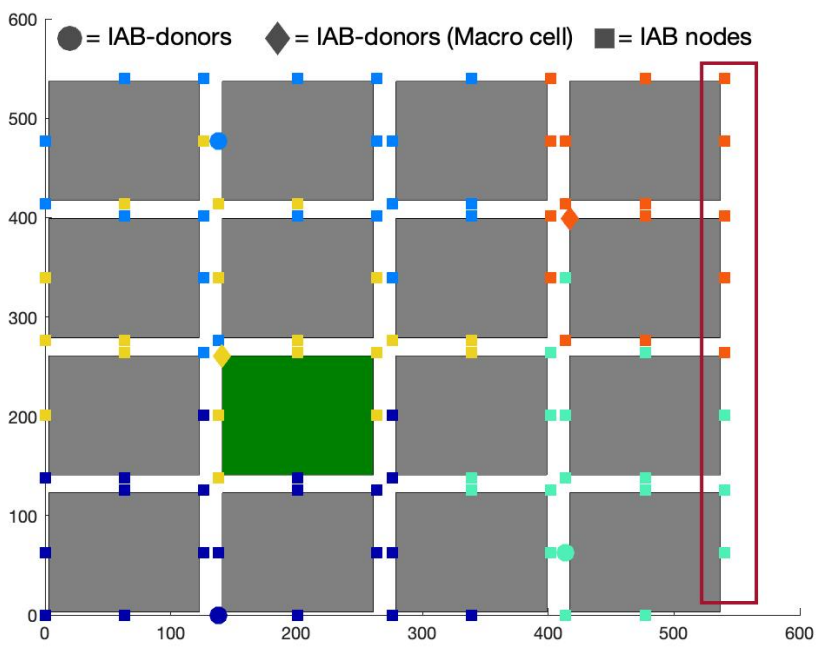

Fig. 5. Partition of the IAB nodes corresponding to the non-uniform traffic demand for the Madrid-grid deployment with 5 IAB-donors, two of which are macrocells (diamond markers). The remaining three donors are shown as circles. Each IAB-donor is shown in a different colour. IAB nodes are shown as coloured squares. The colour indicates the IAB-donor to which an IAB node is associated. IAB nodes in the red rectangle handle twice the traffic demand of the remaining nodes.

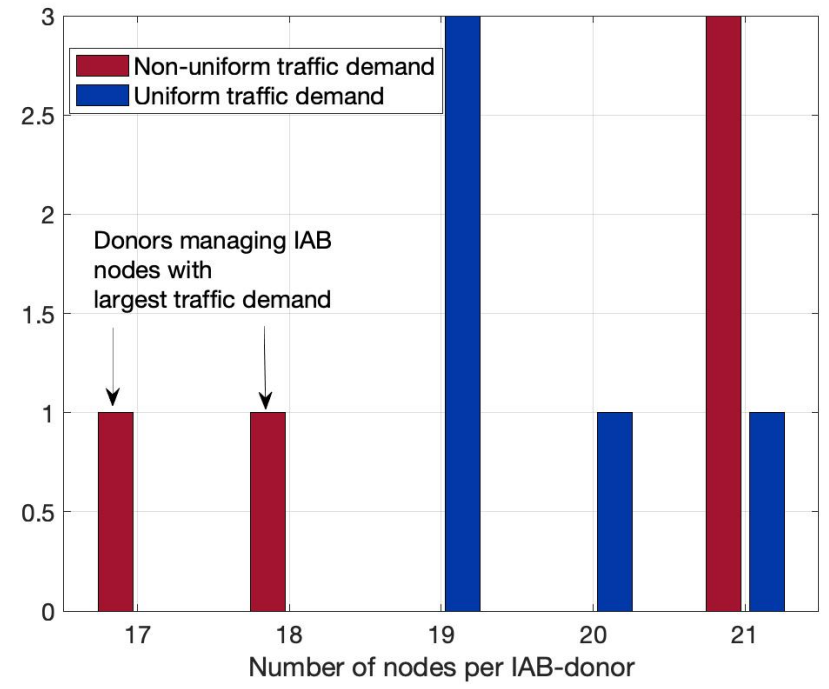

Fig. 6. Distribution of the number of nodes managed by each IAB-donor for the uniform and non-uniform demand scenarios corresponding to the solution of Eq. (3).

a thorough analysis of the proposed formulations taking into account practical considerations, such as the analysis of the delay for the eMBB traffic and the resulting maximum number of hops for the backhaul paths. Our results show that $2 / 3$ of the IAB nodes can reach their donor in 1 or 2 hops (excluding the hop from a UE to the access IAB node). Finally, this work focuses on the case with a single active path and connection to a single IAB donor. Future research can consider the implications of multi-connected IAB nodes and topology changes as nodes connect to two IAB-donors.

\section{REFERENCES}

[1] I. A. Hemadeh, K. Satyanarayana, M. El-Hajjar, and L. Hanzo, "Millimeter-Wave Communications: Physical Channel Models, Design 


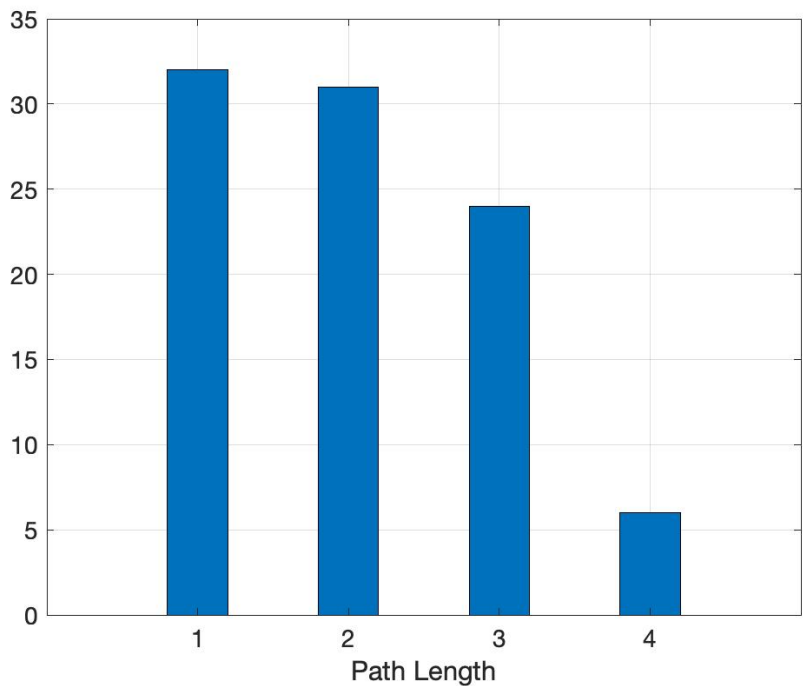

Fig. 7. Histogram of the backhaul path lengths resulting from the minimum path length formulation. Less than $7 \%$ of IAB nodes require 4 hops to reach their IAB-donor.

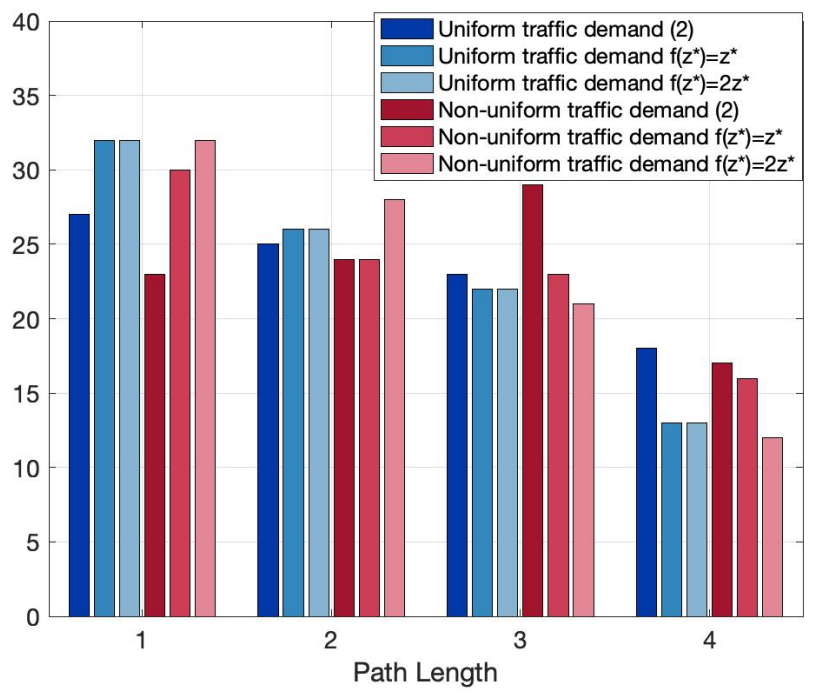

Fig. 8. Comparison of the path length distribution resulting from Eq. (3) and Eq. (11) for uniform and non-uniform traffic demand. A larger value of $f\left(z^{*}\right)$ corresponds to fewer nodes requiring a larger number of hops, both in the case of uniform and non-uniform traffic load.

Considerations, Antenna Constructions, and Link-Budget," IEEE Communications Surveys Tutorials, vol. 20, no. 2, pp. 870-913, 2018.

[2] X. Wang, L. Kong, F. Kong, F. Qiu, M. Xia, S. Arnon, and G. Chen, "Millimeter Wave Communication: A Comprehensive Survey," IEEE Communications Surveys Tutorials, vol. 20, no. 3, pp. 1616-1653, 2018.

[3] 3GPP TR 38.913, "Study on Scenarios and Requirements for Next Generation Access Technologies," Tech. Rep., June 2018.

[4] 3GPP TS 36 300, "Radio Access Network (E-UTRAN); Overall Description; Stage 2 ," Tech. Rep., Jan 2021.

[5] J. Mcmenamy, A. Narbudowicz, K. Niotaki, and I. Macaluso, "HopConstrained mmWave Backhaul: Maximising the Network Flow," IEEE Wireless Communications Letters, vol. 9, no. 5, pp. 596-600, 2020.

[6] C. Madapatha, B. Makki, C. Fang, O. Teyeb, E. Dahlman, M. S. Alouini, and T. Svensson, "On Integrated Access and Backhaul Networks: Current Status and Potentials," IEEE Open Journal of the Communications Society, vol. 1, pp. 1374-1389, 2020.

[7] M. Polese, M. Giordani, A. Roy, D. Castor, and M. Zorzi, "Distributed Path Selection Strategies for Integrated Access and Backhaul at mmWaves," in 2018 IEEE Global Communications Conference
(GLOBECOM), 2018, pp. 1-7.

[8] M. Polese, M. Giordani, T. Zugno, A. Roy, S. Goyal, D. Castor, and M. Zorzi, "Integrated Access and Backhaul in 5G mmWave Networks: Potential and Challenges," IEEE Communications Magazine, vol. 58, no. 3, pp. 62-68, 2020.

[9] M. Simsek, O. Orhan, M. Nassar, O. Elibol, and H. Nikopour, "IAB Topology Design: A Graph Embedding and Deep Reinforcement Learning Approach," IEEE Communications Letters, pp. 1-1, 2020.

[10] M. N. Islam, N. Abedini, G. Hampel, S. Subramanian, and J. Li, "Investigation of Performance in Integrated Access and Backhaul Networks," in IEEE INFOCOM 2018 - IEEE Conference on Computer Communications Workshops (INFOCOM WKSHPS), 2018, pp. 597-602.

[11] 3GPP TS 38.401, "NG-RAN; Architecture description,", Dec 2017.

[12] ITU-R, "Minimum Requirements Related to Technical Performance for IMT-2020 Radio Interface(s), M.2410-0 ”, Nov 2017.

[13] 3GPP, "TR 38.913 Study on Scenarios and Requirements for Next Generation Access Technologies, V15 ," Tech. Rep., Jun 2018.

[14] — "TR 37.910 Study on Self-evaluation towards IMT-2020 Submissions, v16.1 ," Tech. Rep., Sep 2019.

[15] ITU-R, "Initial evaluation report from ATIS WTSC IMT-2020 evaluation group, Document 5D/3-E,", Nov 2019.

[16] J. Mcmenamy, A. Narbudowicz, K. Niotaki, and I. Macaluso, "Hopconstrained mmwave backhaul: Maximising the network flow," IEEE Wireless Communications Letters, vol. 9, no. 5, pp. 596-600, 2020.

[17] J. F. Monserrat and M. Fallgren, "METIS - Simulation Guidelines," FP7-ICT-317669, , Oct 2013.

[18] CST Computer Simulation Technology GmbH, Darmstadt, Germany, "CST-Computer Simulation Technology Microwave Studio." [Online]. Available: https://www.cst.com/products/cstmws

[19] T. S. Rappaport, G. R. MacCartney, M. K. Samimi, and S. Sun, "Wideband Millimeter-Wave Propagation Measurements and Channel Models for Future Wireless Communication System Design," IEEE Transactions on Communications, vol. 63, no. 9, pp. 3029-3056, 2015. 